\title{
Trapping of $\mathrm{Li}^{+}$Ions by $\left[\mathrm{ThF}_{\mathrm{n}}\right]^{4-\mathrm{n}}$ Clusters Leading
}

\section{to Oscillating Maxwell-Stefan Diffusivity in Molten}

\section{Salt $\mathrm{LiF}_{-} \mathrm{ThF}_{4}$}

\author{
Brahmananda Chakraborty $^{{ }^{*}}$, Sharif Kidwai ${ }^{2}$ and Lavanya M. Ramaniah ${ }^{l}$ \\ ${ }^{1}$ High Pressure and Synchrotron Radiation Physics Division, Bhabha Atomic Research Centre, \\ Trombay, Mumbai-400085, India \\ ${ }^{2}$ Department of Electrical Engineering, Aligarh Muslim University, Aligarh-202002, India \\ E-mail address: brahma@barc.gov.in \\ Phone: +91-2225592057
}

\section{THEORETICAL OUTLINE}

Multicomponent diffusion in the present work is computed according to the Maxwell-Stefan (MS) approach, first proposed by Maxwell and extended by Stefan, ${ }^{1}$ where the driving force $(\hat{\mathbf{X}})$ is proportional to the relative velocity between the species in the system. ${ }^{2}$ Here we follow the framework originally developed by Krishna and Wesselingh, ${ }^{2}$ where the driving thermodynamics force can be expressed in terms of molar fluxes relative to the mean molar velocity as

$$
-\frac{x_{i}}{R T} \nabla \mu_{i}=\sum_{\substack{k=1 \\ k \neq i}}^{n} \frac{1}{c \bigoplus_{i k}}\left(x_{k} \mathbf{J}_{i}^{\cdot}-x_{i} \mathbf{J}_{k}\right)
$$


Where molar flux is defined relative to a reference velocity $\hat{\mathbf{V}}^{\bullet}$ as

$$
\hat{\mathbf{J}}_{i}^{\bullet}=c_{i}\left(\hat{\mathbf{U}}_{i}-\hat{\mathbf{V}}^{\bullet}\right)=c x_{i}\left(\hat{\mathbf{U}}_{i}-\hat{\mathbf{V}}^{\bullet}\right) \quad(i=1,2, \ldots, n)
$$

with $x_{i}$ and $c_{i}$ are the mole fraction and molar density of the $i^{\text {th }}$ species respectively. The mean molar velocity in this mole-fraction $(x)$ based formalism is defined as

$$
\hat{\mathbf{V}}^{\bullet}=\sum_{i=1}^{n} x_{i} \hat{\mathbf{U}}_{i}
$$

We can rewrite Eq (1) in matrix form as

$$
\hat{\mathbf{X}}^{\bullet}=\mathbf{B} \hat{\mathbf{J}}^{\bullet}
$$

where $\hat{\mathbf{X}}$ and $\hat{\mathrm{J}}$ are vectors of dimension $(n-1)$ and $\mathbf{B}$ is a $(n-1)$ by $(n-1)$ matrix with the elements of $\mathbf{B}$ given $b^{3}$

$$
B_{i j}=\delta_{i j}\left(\frac{x_{i}}{\bigoplus_{i n}}+\sum_{\substack{k=1 \\ k \neq i}}^{n} \frac{x_{k}}{\bigoplus_{i k}}\right)+\left(1-\delta_{i j}\right) x_{i}\left(\frac{1}{\bigoplus_{i n}}-\frac{1}{\bigoplus_{i j}}\right)
$$

and $\delta_{i j}$ is the Kronecker delta function.

We have employed the concepts of linear phenomenological theory, according to which the diffusive fluxes in an $n$-component system are linear homogeneous functions of thermodynamic forces as given by ${ }^{4,5}$

$$
\hat{\mathbf{J}} \equiv \Lambda \hat{\mathbf{X}}
$$

where $\hat{\mathbf{J}}$ and $\hat{\mathbf{X}}$ are the diffusive flux and thermodynamic force vectors, respectively, and $\boldsymbol{\Lambda}$ is the Onsager matrix containing the phenomenological coefficients. 
Now we will write the volume-averaged diffusive flux ${ }^{6}$ which is simply the sum of the atom velocities relative to the barycentric velocity (constrained to be zero), with details given in earlier work $^{7}$ as

$\Im_{k}^{0}(t)=\int_{\Omega} \mathbf{j}_{k}(\mathbf{r}, t)=\sum_{l=1}^{N_{k}}\left[\mathbf{u}_{k}^{l}(t)-\mathbf{V}^{0}(t)\right]$

where $l$ denotes the atom/ion index and $N_{k}$ is the total number of atoms/ions for the $k^{\text {th }}$ species, $V^{0}$ is the velocity of centre of the mass of the system and superscript 0 signifies the barycentric reference frame. Using the Green Kubo formulas the Onsager coefficients can be computed through MD simulations as time correlations of flux fluctuations ${ }^{6}$ and the elements of the Onsager dynamical matrix can be expressed as

$\Lambda_{i k}^{0}=\frac{1}{3 N} \int_{0}^{\infty} d t\left\langle\Im_{i}^{0}(t) \Im_{k}^{0}(0)\right\rangle \equiv \frac{1}{3 N} \int_{0}^{\infty} d t \Psi_{i k}^{0}(t)$

where $N$ is the total number of atoms/ions in the system, and $\Psi(t)$ indicates the ensemble average of the diffusive flux correlation which can give insight regarding dynamics of the system. We can also compute the Onsager matrix elements using atomic positions at various time steps using Einstein form which is equivalent to the Green-Kubo form in Eqn. (8) and can be expressed as ${ }^{8}$

$\Lambda_{i k}^{0}=\frac{1}{6 N} \lim _{t \rightarrow \infty} \frac{d}{d t}\left\langle\left[\mathbf{r}_{i}^{0}(t)-\mathbf{r}_{i}^{0}(0)\right] \cdot\left[\mathbf{r}_{k}^{0}(t)-\mathbf{r}_{k}^{0}(0)\right]\right\rangle$

Now we will outline how MS diffusivity can be obtainded from the elements of Onsager dynamic matrix computed through MD simulations in barycentric reference frame using either Green Kubo form or Einstein form. We can write Eq (4) as

$\hat{\mathbf{J}}^{\bullet}=\mathbf{B}^{-1} \hat{\mathbf{X}}^{\bullet} \equiv \hat{\mathbf{\Lambda}}^{\bullet} \cdot \hat{\mathbf{X}}^{\bullet}$ 
A comparison of Equ.(6) and Equ. (10) leads to the inference that the matrix $\mathbf{B}^{-1}$ is equivalent to Onsager matrix obtained through MD simulations. It is to be noted that in Equ.(10) the Onsager matrix $\boldsymbol{\Lambda}$ is in mean molar velocity frame whereas computed Onsager matrix through MD simulations is in barycentric referrence frame with $n \times n$ Onsager coefficients. After appropriate transformation of the reference frame and dimension reduction from $n$ to $(n-1)$, the matrix elements $\mathbf{B}^{-1}(=\mathbf{K})$ for a ternary system can be expressed in terms of Onsager coefficients as ${ }^{3}$

$$
\begin{aligned}
& K_{11}=\left(1-x_{1}\right)\left(\frac{\Lambda_{11}}{x_{1}}-\frac{\Lambda_{13}}{x_{3}}\right)-x_{1}\left(\frac{\Lambda_{21}}{x_{1}}-\frac{\Lambda_{23}}{x_{3}}+\frac{\Lambda_{31}}{x_{1}}-\frac{\Lambda_{33}}{x_{3}}\right) \\
& K_{12}=\left(1-x_{1}\right)\left(\frac{\Lambda_{12}}{x_{2}}-\frac{\Lambda_{13}}{x_{3}}\right)-x_{1}\left(\frac{\Lambda_{22}}{x_{2}}-\frac{\Lambda_{23}}{x_{3}}+\frac{\Lambda_{32}}{x_{2}}-\frac{\Lambda_{33}}{x_{3}}\right) \\
& K_{21}=\left(1-x_{2}\right)\left(\frac{\Lambda_{21}}{x_{1}}-\frac{\Lambda_{23}}{x_{3}}\right)-x_{2}\left(\frac{\Lambda_{11}}{x_{1}}-\frac{\Lambda_{13}}{x_{3}}+\frac{\Lambda_{31}}{x_{1}}-\frac{\Lambda_{33}}{x_{3}}\right) \\
& K_{22}=\left(1-x_{2}\right)\left(\frac{\Lambda_{22}}{x_{2}}-\frac{\Lambda_{23}}{x_{3}}\right)-x_{2}\left(\frac{\Lambda_{12}}{x_{2}}-\frac{\Lambda_{13}}{x_{3}}+\frac{\Lambda_{32}}{x_{2}}-\frac{\Lambda_{33}}{x_{3}}\right)
\end{aligned}
$$

Now using Equ. (5) the three independent MS diffusivities for a ternary mixture can be expressed in terms of matrix element of $\mathbf{B}$ as

$$
\begin{aligned}
& \mathrm{Ð}_{12}=\left[B_{11}-\left(1+\frac{x_{3}}{x_{1}}\right) B_{12}\right]^{-1} \\
& \mathrm{Ð}_{23}=\left[B_{22}+\left(\frac{x_{1}}{x_{2}}\right) B_{21}\right]^{-1} \\
& \bigoplus_{13}=\left[B_{11}+\left(\frac{x_{2}}{x_{1}}\right) B_{12}\right]^{-1}
\end{aligned}
$$

We have first computed the elements of Onsager matrix $\Lambda$. Then we haved used Equ. (11) to obtain matrix elements of $\mathbf{B}$ from $\mathbf{K}^{-1}$ followed by evaluation of MS diffusivities using Eqn. (12). 


\section{REFERENCES AND NOTES}

1. Curtiss, C. F.; Bird, R. B. Multicomponent Diffusion. Ind. Eng. Chem. Res. 1999, 38, 2515.

2. Krishna, R.; Wesselingh, J. A. The Maxwell-Stefan approach to mass transfer. Chem. Eng. Sci. 1997, 52, 861-911.

3. Krishna, R.; Baten, J. M. V., A. The Darken Relation for Multicomponent Diffusion in Liquid Mixtures of Linear Alkanes: An Investigation using Molecular Dynamics(MD) Simulations. Ind. Eng. Chem. Res. 2005, 44, 6939-6947.

4. Groot, S. R. D.; Mazur, P. Nonequilibrium Thermodynamics ; Dover Publications Inc.: New York, 1984.

5. Hanley, H. J. M. Transport Phenomena in Fluids; Marcel Dekker: New York, 1969.

6. Wheeler, D. R.; Newman, A. Molecular dynamics simulations of multicomponent diffusion. J. Phys. Chem. B , 2004, 108, 18353-18361.

7. Chakraborty, B.; Wang J.; Eapen, J.; Multicomponent diffusion in molten LiCl-KCl: Dynamical correlations and divergent Maxwell-Stefan diffusivities, Phys. Rev. E 2013, $87,052312$.

8. Keffer, J.; Gao, C.Y.; Edwards, B., A. On the Relationship between Fickian Diffusivities at the Continuum and Molecular Levels, J.Phys. Chem. B, 2005, 109, 52795288. 